\title{
Images contain what the imager put there: A nonreplication of illusions in imagery
}

\author{
DANIEL REISBERG and ANNE MORRIS \\ New School for Social Research, New York, New York
}

\begin{abstract}
Although much research points to the commonalities between imaging and perceiving, there are also large differences between these activities. Perception begins with a distal stimulus; thus, it is possible to ask whether a percept corresponds to its stimulus, implying the possibility of nonveridicality or of ambiguity. In contrast, images come into being accompanied by an understanding of what the image represents; hence, images cannot be misunderstood, and they represent what the imaginer intended. Some recent results showing illusions in imagery appear to undermine these claims, but we argue that these data are potentially flawed. We report results that are consistent with these claims about the nature of images and the relation between imagery and perception.
\end{abstract}

There have been many demonstrations that visual perception and visual imagery share a number of properties (Farah, 1985; Finke, 1980; Kosslyn, 1980; Shepard \& Cooper, 1983). However, there also are large differences between these two activities, and we believe that the implications of these differences have not been explored adequately. One potentially large contrast between imagery and perception is that the latter begins with a distal stimulus and the former does not. Thus, it is possible to ask whether a percept corresponds to its stimulus, implying the possibility of nonveridicality or of ambiguity. Images, on the other hand, typically begin with an intent. As a number of authors have argued (Casey, 1976; Fodor, 1981; Kolers, 1983; Kolers \& Smythe, 1984; Reed, 1974), this concept implies that the image "embodies", that intent. Thus, the image cannot be nonveridical, cannot be misunderstood, and cannot be ambiguous.

Some recent data support one aspect of this view, namely the claim about ambiguity. Chambers and Reisberg (1985) asked subjects to encode drawings into memory. Subjects then were asked to image the drawing which was, in fact, one of the classical ambiguous figures, and they were asked to inspect that image for an interpretation other than the one initially seen. No subject in these procedures discovered the alternate construal of an imaged "ambiguous" figure. In contrast, a moment later, all of the subjects were able to draw a picture of their image, and then to discover the other construal in their own drawings.

These findings support the claim that images come into being accompanied by an understanding both of what the image represents and of how its appearance is to be or-

This research was supported by funds from the New School for Social Research. We wish to thank Peter Monk for his discussion of the analysis. Address correspondence to: D. Reisberg, Department of Psychology, New School for Social Research, 65 Fifth Avenue, New York, NY 10003. ganized (i.e., what is figure, what is ground, where is the "top," etc.). Although these results support one aspect of this more general view, other recent data appear to contradict this view; in particular, some recent data seem to show that images can be nonveridical. Wallace (1984a, 1984b) showed subjects line drawings and asked them to imagine that certain other lines also were present. The composite drawing was a reproduction of the configuration of a standard illusion. Wallace then asked whether subjects reported an illusion with these hybrid figures. For example, subjects were shown the two converging lines and one of the horizontals of the Ponzo figure; subjects then were instructed "to imagine a line of the same physical length as the horizontal one present and to imagine their line as being located below the one that they could see" (Wallace, 1984a, p. 157).

Wallace $(1984 a, 1984 b)$ reported that subjects with vivid imagery (according to a self-report questionnaire) consistently reported an illusion with these figures. Subjects with less vivid imagery reported robust illusions when the lines were physically present, but no illusion with imaged figures.

These data appear to undermine the view of imagery offered above. If perception and imagery are equivalent in producing illusions, then subjects "misread" their own images. Alternatively, but also incompatible with our view of imagery, the illusion may arise in the creation of the image: Subjects intended to create an image specified as having a particular appearance and representing a particular state of affairs (i.e., equal lines). Despite this, subjects ended up with an image different from this specification, suggesting that, in this circumstance at least, the contents of an image can depart from the imaginer's understanding or intention.

One might object to Wallace's (1984a, 1984b) data on the grounds that subjects may have responded to some social cue in the experimental situation, so that we are simply seeing a case of experimenter demand. It also is 
conceivable that subjects guessed the correct answers in these tasks, perhaps by using some rudimentary sense of the rules of perspective. These possibilities seem credible since, in the subjects' view, the task was a confusing one: Subjects were told, first, to imagine equal lines but were asked a moment later to determine whether or not the lines were equal. In this situation, subjects might well have sought any cue or strategy that would help them respond to this seemingly peculiar task.

Wallace $(1984 a, 1984 b)$ offered what appears to be a persuasive response to these challenges, but his response does not stand up to scrutiny. He noted that demand characteristics or guessing might have led subjects to the illusion response with these figures, but argued that these factors were equivalent for high-vividness and lowvividness imagers. The fact that the image illusions occurred only for the high-vividness imagers suggests that these artifacts were not responsible for the result. Instead, the dependence of the effect on vivid imagery argues that, as claimed, subjects experienced an imaged visual illusion.

The flaw in this argument lies in its premise: the suggestion that these extraneous factors were equivalent for high- and low-vividness subjects. In all of Wallace's (1984a, 1984b) studies, subjects were asked whether they were "able to see" the line(s) specified by the experimenter, whether they were able to "produce" or "conjure up" the relevant line. If the subject responded "yes" to this question, he/she was asked about the relative lengths of the figure's lines; if the subject responded "no" to the question, the procedure ended. Wallace reported that virtually all of the high-vividness imagers did answer "yes," and so continued through the entire procedure. Virtually none of the low-vividness imagers answered "yes" to the question. Consequently, we cannot assume that the demand characteristics were the same for high- and low-vividness imagers, because one group was exposed to only half of the procedure. Worse, we cannot rest any claims on the data contrast between the two groups, as the critical data are available for only the high-vividness imagers; we have no way of knowing how the low-vividness imagers would have responded if asked about line lengths.

A replication of these studies seems appropriate. The procedure to be described is matched to Wallace's (1984a), with three changes. First, all subjects were required to complete the procedure to provide a means of comparing high- and low-vividness imagery subjects. Second, to minimize any demand effects, the procedure was run as a questionnaire. In our study, the experimenter was in the room while the subject filled out the questionnaire, but there was no interaction between them during the procedure. Finally, we attempt to confound any guessing strategy subjects might try to use: Subjects were asked about several different figures, all variants on the Ponzo illusion. Some of these were modified in ways known to be irrelevant to the illusion; some were modified in ways known to eliminate the illusion. We reasoned that subjects were unlikely to guess which of these modifications would matter; hence, any guessing strategy would be unsuccessful in reproducing the standard perceptual illusion results.

\section{METHOD}

\section{Subjects}

Eighteen subjects were recruited from an introductory psychology course at a community college. The course did not include discussion of perceptual illusions.

\section{Stimuli and Procedure}

Subjects were asked to complete a brief questionnaire that contained a section asking them about imaged illusions, a section asking them about actual illusions, and a final section that assessed imagery vividness.

Our first figure was the standard Ponzo illusion (Figure 1A). For the perception trials, all four of the line segments were presented (i.e., both oblique lines and both horizontal lines); for the imagery trials, the lower horizontal line was replaced by a single dot located where the line's center would have been. The second figure was a control figure: the oblique lines were presented in the standard orientation, but the two horizontal segments were replaced by two vertical segments (Figure 1B). We expected this configuration to produce no illusion (cf. Coren \& Girgus, 1978). The lower segment was replaced by a dot for the imagery trials. Our third and fourth figures were modified versions of the first and second; these figures were rotated $90^{\circ}$, and the inducing obliques were replaced with dashed lines (Figures 1C and 1D). Neither of these modifications affects the illusion (Coren \& Girgus, 1978).

All figures were drawn in black ink on white paper. The oblique lines were $80 \mathrm{~mm}$ in length, separated by an angle of $60^{\circ}$. The enclosed lines were $23 \mathrm{~mm}$ in length. In Figures $1 \mathrm{~A}$ and $1 \mathrm{C}$, the enclosed lines were 23 and $50 \mathrm{~mm}$ from the apex of the obliques; in Figures 1B and 1D, the distances were 17 and $48 \mathrm{~mm}$.

In the first section of the questionnaire, subjects were instructed to image an additional line into each figure shown. For example, the instructions for the imaginal version of Figure 1A told the subject to "imagine a horizontal line equal in length to the horizontal line already shown, with the center of the new line located on the dot in the figure." Subjects then were asked whether they were able to "see the line" described; a 0-7 response scale was provided, with 0 marked "I can see it as if it was really there" and 7 marked "I'm thinking about it but in no way do I feel like I'm seeing it." Subjects then were asked whether the imagined line appeared longer than, equal to, or shorter than the other horizontal line (with order of these alternatives randomized). Next, subjects were asked how they knew, with alternatives ranging from "I have no idea; I'm guessing" to "I have a mental picture of the figure and I can see in my picture exactly how much the two lines do (or do not) differ." On the next page, subjects were shown a horizontal line $(8 \mathrm{~cm}$ long) and were instructed to mark off the perceived difference between
$1 \mathrm{~A}$
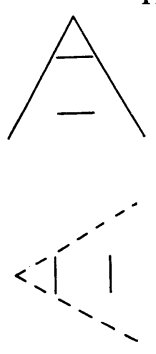

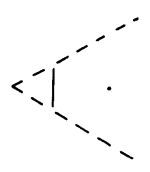

1C

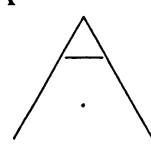

1B
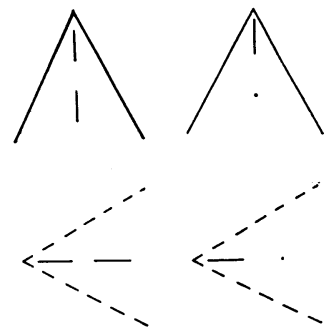

1D
Figure 1. The four pairs of figures used as experimental stimuli. Figures $1 \mathrm{~A}$ and $1 \mathrm{C}$ are the illusion figures; $1 \mathrm{~B}$ and $1 \mathrm{D}$ are the control figures. Within each pair, the left figure was used in the perception condition, and the right figure in the imagery condition. 
the target lines. (Subjects were told to skip this step if they had responded that the lines appeared equal in length.)

The perception questions always followed the imagery questions; subjects were not allowed to return to the imagery questions once they began the perception section. The format for this section was the same as for the imagery section, except that the "can you see the line" and "how do you know" questions were omitted. As before, the horizontal line on which subjects marked the magnitude of the difference was on the page following the illusion.

Finally, the subject filled out a written-response version of Marks's Vividness of Visual Imagery Questionnaire (VVIQ), which employs a 7 -point response scale in place of the usual 5 , and makes the 0 response indicate low imagery, rather than high.

\section{RESULTS}

The main results are shown in Table 1. First, when confronted with the actual stimulus, our subjects were vulnerable to the illusions. Subjects tended to make responses consistent with the illusion when looking at the experimental figures; when looking at the control figures, they tended to respond that the enclosed lines were equal. This difference between experimental and control figures is significant for both the standard illusion and the rotated version $\left(\chi^{2}=8.10\right.$ and 7.11 , respectively, both ps $\left.<.01\right)$. Second, subjects were not vulnerable to the illusions when confronted with the imagined versions of these figures; the difference between experimental and control figures yielded $\chi^{2}=0$ for the standard figure and $\chi^{2}=.17$ for the rotated figure. (These analyses, using the McNemar procedure for related samples, simply dichotomize the data into success and failure reponses-in our case illusion and nonillusion.) Third, a small number of subjects in the imagery condition gave responses consistent with the illusion, but these were not associated with particularly high imagery; three-quarters of these responses came from subjects with VVIQ scores below the group's median. These responses also were no more likely when subjects claimed to "see" the imagined line. If we consider only trials in which subjects marked " 3 " or lower on this scale, there were four "illusion" responses, five "equal" responses, and seven "anti-illusion" responses. If we use " 2 "' as the cut-off, the frequencies were two, four, and four, respectively. The result is also the same if we divide the data on the basis of our "how do you know" question. A " 4 " response on this Likert scale corresponded to asserting "I have a mental picture of the figure (including the added line) and I can see the relative lengths of the two lines in my mental picture." Subjects who gave this or a stronger judgment generated five illusion responses, nine judgments of "equal," and five responses opposed to the illusion. In all of these ways, the data from "imagery" responses are the same as those in the overall pattern, and show no illusion.

Finally, although the imagery condition does not induce the standard illusion, there is nonetheless a difference between the imagined illusion (Figures $1 \mathrm{~A}$ and $1 \mathrm{C}$ ) and control figures (Figures 1B and 1D). Subjects tended to give "equal" responses to the latter, but more evenly dispersed responses to the former. If we dichotomize the responses into "equal" and "unequal," there is a reliable difference between illusion and control responses $\left(\chi^{2}=4.17\right.$, $\mathrm{p}<.05$, and $\chi^{2}=7.36, \mathrm{p}<.01$, for the imaged standard and imaged rotated figures, respectively). (We return to this point below.)

Perhaps our sample included an insufficient number of subjects with truly vivid imagery. Our subjects ranged from 2.88 to 6.25 on the VVIQ's 7-point response scale, with a mean of 5.17 . Half of the 18 subjects responded that their images were at least "moderately clear and vivid" (a response of 5). Hence, we see no basis for claiming that our subjects did not have adequately vivid visual imagery.

\section{DISCUSSION}

In our procedure, subjects who imagined the configuration of a standard geometric illusion did not yield a reliable illusory effect. This was true for both subjects with vivid visual imagery and subjects without vivid imagery, results that are in clear contrast to data reported by Wallace (1984a, 1984b)

Even if there was no reliable imagery illusion, subjects in the imagery condition still responded differently to the illusion and control figures. More specifically, if we are correct that images embody intents, then subjects following an instruction to imagine equal lines should have imagined just that. Why did subjects imaging Figures $1 \mathrm{~A}$ and $1 \mathrm{C}$ not prefer the "equal" response? We would argue that this simply reflects subjects' guessing strategies. Subjects in this procedure were first told to imagine equal lines, and then were asked whether the lines were equal. As we noted earlier, subjects may quite naturally suspect that this is a "trick question"' and try to discover the trick. There are various factors that might lead subjects to believe that the trick concerns the illusion figures (e.g., an intuitive sense that one should consider the bounds to horizontal extent, prominent in Figure 1A but not in Figure 1B). Given this supposition plus a diversity of guessing strategies, one would predict the dispersion of responses we have observed. We have little stake,

Table 1

Response Frequencies

\begin{tabular}{|c|c|c|c|c|c|c|}
\hline \multirow[b]{2}{*}{ Stimulus } & \multicolumn{3}{|c|}{$\begin{array}{c}\text { Perception Condition } \\
\text { (both test lines present) }\end{array}$} & \multicolumn{3}{|c|}{$\begin{array}{c}\text { Imagery Condition } \\
\text { (one test line present, } \\
\text { one imagined) }\end{array}$} \\
\hline & $\begin{array}{l}\text { Top > } \\
\text { Bottom }\end{array}$ & $\begin{array}{l}\text { Top }= \\
\text { Bottom }\end{array}$ & $\begin{array}{l}\text { Top }< \\
\text { Bottom }\end{array}$ & $\begin{array}{l}\text { Top > } \\
\text { Bottom }\end{array}$ & $\begin{array}{l}\text { Top }= \\
\text { Bottom }\end{array}$ & $\begin{array}{l}\text { Top < } \\
\text { Bottom } \\
\end{array}$ \\
\hline Standard Illusion & 11 & 6 & 1 & 6 & 5 & 7 \\
\hline Control Figure & 1 & 17 & 0 & 5 & 11 & 2 \\
\hline Rotated Illusion & 12 & 4 & 2 & 6 & 4 & 8 \\
\hline Rotated Control & 3 & 14 & 1 & 4 & 13 & 1 \\
\hline
\end{tabular}

Note - "Top" refers to the line closer to figure's apex; "bottom" refers to the line further from it. In the rotated figure, "top" and "bottom" were on the left and right, respectively. 
however, in the details of this account. What is important is that, in the imagery condition, responses opposite to the illusion are just as likely as responses consistent with the illusion. Thus, whatever extraneous factors are producing this pattern, they are clearly distinct from the factors underlying the perceptual illusion.

Thus, we conclude that prior demonstrations of imagery illusions are attributable either to guessing or to demand effects. When these are controlled, no illusion remains. This removes what appeared to be a serious obstacle for the claims we are trying to develop. If an image is created to represent a state of affairs, then it represents them. The representation may be a fair likeness or a poor one, with details specified or left indeterminate, but, in any of these cases, it unambiguously represents that which was intended (cf. Chambers \& Reisberg, 1985). Likewise, as we have tried to show here, images are defined by our understanding of them, and there can be no possibility of a nonveridical reading of the image.

We do not mean for these claims to deny the important commonalities between imagery and perception, such as those documented by Farah (1985), Finke (1980), and others. Our intent is to argue that these commonalities stand alongside important differences between imagery and perception. Until the functional implications of the contrasts are explored, we are in no position to assess the overall similarity between these activities.

Finally, throughout this paper, we have talked about "imagery" or "imagination" conditions when, in fact, the relevant conditions, following Wallace (1984a, 1984b), involve hybrid stimuli: some of the lines were imagined and some actually were perceived. Such hybrids are probably quite common in our day-to-day imagining. It seems likely that one important function of imagery is to allow anticipation of how things will look, and a great deal of this anticipation builds on alreadypresent objects or scenes. We imagine how some object will appear in a context while looking at that context; for example, standing in one's room, one tries to imagine how a new chair will fit. We also try to imagine contexts onto targets, for example, asking oneself whether the stone in one's hand is the right shape for the gap in the fireplace under construction.

It is far from clear to us just how these hybrids will behave, and thus how well we can anticipate perception. Our argument in the present paper has been that some of these anticipations are inaccurate, and we presume there are many such cases. Nonetheless, we believe that we frequently can use imagination to anticipate how things will look. It is interesting in this regard to consider the case opposite to the one examined here. We have argued that perceived context should not lead one to misread imagined targets. Can imaged context cause us to misperceive actually present lines? We have held this case aside in the present study, because it does not bear directly on our claims about imagery. If this illusory effect exists, then it is perceived lines that are misjudged, and thus no misreading of images is entailed. Likewise, the presence of the illusion does not imply a difference between one's image and one's intent, since the image will represent just what it should, that is, the inducing context.

Wallace (1984a, 1984b) and Berbaum and Chung (1981) have reported illusions from imagined contexts, but the data are uncompelling. The Wallace results are subject to the same argument as offered above. Berbaum and Chung asked subjects to "create" the Müller-Lyer figure by imagining the "fins" onto the to-be-judged lines. The figure actually shown contained the target lines and dots, positioned where the fins' end-points would normally be; subjects were instructed to image the connecting lines between the dots and the lines shown. However, no steps were taken to rule out demand artifacts. In addition, this effect may not depend on the imaged lines. We know that illusions of size can be induced by manipulating the size of the area which surrounds the target (Coren \& Girgus, 1978). It seems possible, then, that the Berbaum and Chung figure (a variant of the figure used by Coren \& Porac, 1983; Kohler, 1947) will produce the illusion without any supplements from imagery.

Hence we regard it as an open issue whether these procedures do or do not yield illusions, and hence whether there is an asymmetry between imagery illusions, induced by perception, and perceptual illusions, induced by imagery. We have argued that the former should not exist, but our account of imagery does not rule out the latter. Given the suggested functionality of these hybrid figures, this seems an interesting place for future research.

\section{REFERENCES}

Berbaum, K., \& ChUng, C. (1981). Müller-Lyer illusion induced by imagination. Journal of Mental Imagery, 5, 125-128.

CASEY, E. (1976). Imagining: A phenomenological study. Bloomington, IN: Indiana University Press.

Chambers, D., \& Reisberg, D. (1985). Can mental images be ambiguous? Journal of Experimental Psychology: Human Perception \& Performance, 11, 317-328.

Coren, S., \& GiRgus, J. (1978). Seeing is deceiving: The psychology of visual illusions. Hillsdale, $\mathrm{NJ}$ : Erlbaum.

CoRen, S., \& PORAC, C. (1983). The creation and reversal of the MüllerLyer illusion through attentional manipulation. Perception, 12, 49-54.

FARAH, M. (1985). Psychophysical evidence for a shared representational medium for mental images and percepts. Journal of Experimental Psychology: General, 114, 91-103.

FINKE, R. (1980). Levels of equivalence in imagery and perception. Psychological Review, 87, 113-132.

FoDOR, J. (1981). Imagistic representation. In N. Block (Ed.), Imagery (pp. 63-86). Cambridge, MA: MIT Press.

KoHLER, W. (1947). Gestalt psychology. New York: Liveright.

Kolers, P. (1983). Perception and representation. Annual Review of Psychology, 34, 129-166.

Kolers, P., \& SMYTHE, W. (1984). Symbol manipulation: Alternatives to the computational view of mind. Journal of Verbal Learning \& Verbal Behavior, 23, 289-314.

KossLYN, S. (1980). Image and mind. Cambridge, MA: Harvard University Press.

REED, S. (1974). Structural descriptions and the limitations of visual images. Memory \& Cognition, 2, 329-336.

SHEPARD, R., \& COOPER, L. (1983). Mental images and their transformations. Cambridge, MA: MIT Press.

WALLACE, B. (1984a). Apparent equivalence between perception and imagery in the production of various visual illusions. Memory \& Cognition, 12, 156-162.

WALLACE, B. (1984b). Creation of the horizontal-vertical illusion through imagery. Bulletin of the Psychonomic Society, 22, 9-11.

(Manuscript received for publication August 22, 1985.) 